\title{
A divergent spirochete strain isolated from a resident of the southeastern United States was identified by multilocus sequence typing as Borrelia bissettii.
}

Maryna Golovchenko ${ }^{1,2}$, Marie Vancová ${ }^{1}$, Kerry Clark³, James H. Oliver Jr ${ }^{2}$, Libor Grubhoffer ${ }^{1,4}$ and Nataliia Rudenko ${ }^{1,2^{*}}$

\begin{abstract}
Background: Out of 20 spirochete species from Borrelia burgdorferi sensu lato (s.l.) complex recognized to date some are considered to have a limited distribution, while others are worldwide dispersed. Among those are Borrelia burgdorferi sensu stricto (s.s.) and Borrelia bissettii which are distributed both in North America and in Europe. While B. burgdorferi s.s. is recognized as a cause of Lyme borreliosis worldwide, involvement of B. bissettii in human Lyme disease was not so definite yet.

Findings: Multilocus sequence typing of spirochete isolates originating from residents of Georgia and Florida, USA, revealed the presence of two Borrelia burgdorferi sensu stricto strains highly similar to those from endemic Lyme borreliosis regions of the northeastern United States, and an unusual strain that differed from any previously described in Europe or North America. Based on phylogenetic analysis of eight chromosomally located housekeeping genes divergent strain clustered between Borrelia bissettii and Borrelia carolinensis, two species from the B.burgdorferi s.l. complex, widely distributed among the multiple hosts and vector ticks in the southeastern United States. The genetic distance analysis showed a close relationship of the diverged strain to B. bissettii.

Conclusions: Here, we present the analysis of the first North American human originated live spirochete strain that revealed close relatedness to $B$. bissettii. The potential of B. bissettii to cause human disease, even if it is infrequent, is of importance for clinicians due to the extensive range of its geographic distribution.
\end{abstract}

Keywords: Borrelia, Borrelia bissettii, MLST analysis, live spirochete, divergent strain

\section{Background}

The Borrelia burgdorferi sensu lato (s.l.) complex is a diverse group of bacteria widely distributed in temperate regions of the Northern Hemisphere. From the time of the discovery of the causative agent of Lyme borreliosis (LB), a large number of Borrelia isolates has been obtained from various vertebrate species, including humans. To date, five of the genospecies from B. burgdorferi s.l. complex are ensured human pathogenic,

\footnotetext{
* Correspondence: natasharudenko@hotmail.com

'Biology Centre Czech Academy of Sciences, Institute of Parasitology, Ceske Budejovice 37005, Czech Republic

${ }^{2}$ The James H. Oliver Jr. Institute for Coastal Plain Sciences, Statesboro,

Georgia Southern University, Georgia 30460-8056, USA

Full list of author information is available at the end of the article
}

including $B$. burgdorferi sensu stricto, $B$. afzelii, B. garinii, $B$. bavariensis, and B. spielmanii. Occasionally, $B$. lusitaniae and $B$. kurtenbachii have been detected in patients [1-7]. The recognition of $B$. bissettii as a causative agent of human LB to date was supported by rare cases of molecular detection of B. bissettii DNA in samples of human origin [8-10]. Detection of sequences highly similar to B. bissettii strain DN127 in cardiac valve tissue of a patient with endocarditis and aortic valve stenosis [8], and in serum samples of patients in the Czech Republic [9], raised concern about the pathogenic potential of $B$. bissettii. More recently, the first molecular evidence of infection of 3 out of 27 (11\%) studied residents of Mendocino County (California, USA) with a B. bissettii- 
like spirochete highly similar to strain DN127 further suggested that this species is able to infect humans although LB symptoms were only reported in individuals that had concurrent B. burgdorferi infections [10].

\section{Findings \\ Methods \\ General analysis of Borrelia isolates}

Total DNA was purified from pelleted spirochetes of 3rd passage, cultured from plasma of three residents of southeastern USA [11] in modified Kelly-Pettenkofer (MKP) media [12]. Plasma samples (samples M6p and M11p from Georgia; sample M7p from Florida) were collected earlier for microbiologic testing as part of ongoing studies of tick-borne diseases in the southern United States (UNF IRB approval \#468310-3), but not for the purpose of this study. Written informed consent was obtained from each patient prior to enrolment into the study.

DNA purification, sequencing and sequence analysis were conducted according to protocols established in our laboratory [11, 13]. MLST analysis was conducted according to the scheme developed by Margos and colleagues [14]. Briefly, total DNA from cultured spirochetes was purified using the DNeasy Blood and Tissue kit strictly according to manufacturer's protocol (Qiagen, USA). The MasterTaq Kit (Eppendorf, Germany) with 5x TaqMaster ${ }^{\circ}$ PCR enhancer was used for amplification of eight housekeeping genes using previously described nested primers for $c l p A, c l p X, p e p X, \operatorname{rec} G, r p l B$ and $u v r A$ and semi-nested primers for nifS and $r p l B$ under PCR conditions described earlier [14]. The purified PCR products of expected size were submitted for direct sequencing to the Genomic Laboratory of the Biology Centre Czech Academy of Sciences (Ceske Budejovice, Czech Republic). Sequencing was conducted in both directions, using the same primers that were used for amplification of each locus.

\section{Phylogenetic analysis}

Multiple alignments of the concatenated sequences of the genes $\operatorname{clp} A, \operatorname{clpX}$, nifS, pepX, pyrG, recG, rplB and $u v r A$ were generated in MEGA5 [15]. Sequences of 20 LB group species were downloaded from the MLST database (http://pubmlst.org/borrelia/). A maximum likelihood tree was constructed in MEGA5 using default settings and 500 bootstrap repeats. The general time reversible model was selected. A genetic distance analysis was conducted in MEGA5 using the Kimura 2parameter model [16] and the concatenated sequences of housekeeping loci. The rate variation among sites was modelled with a gamma distribution (shape parameter = 0.6). The analysis involved 29 nucleotide sequences (four B. carolinensis, one B. americana, one B. kurtenbachii, thirteen B. bissettii and seven B. burgdorferi sequence types (STs) and the three strains analysed in this study). Codon positions included were $1 \mathrm{st}+2 \mathrm{nd}+3 \mathrm{rd}+$ Noncoding. All positions containing gaps and missing data were eliminated. There were a total of 4785 positions in the final dataset. The evolutionary analysis was conducted in MEGA6 [17].

\section{Results}

\section{Multilocus sequence typing and phylogenetic analysis}

MLST of spirochete isolates originating from residents of Georgia and Florida, USA, revealed the presence of two B. burgdorferi sensu stricto strains, named M6p and M11p, highly similar to those from the northeastern United States, and an unusual strain, M7p, that differed from any previously described in Europe or North America.

Results of MLST analysis showed that samples M6p and M11p (indicated by $\boldsymbol{\Lambda}$, Fig. 1) clustered within the B. burgdorferi s.s. clade (Fig. 1). Strain M6p was found close to ST11, and strain M11p was positioned between ST58 and ST59. The affiliation of these samples to the species $B$. burgdorferi s.s. was confirmed by a genetic distance analysis (Table 1); the values for genetic distance were very low $(<=0.004)$ to all $B$. burgdorferi STs included in the analysis, even those that did not represent next neighbors in the tree, e.g. B31, ST23, ST22. The number of variable sites to the closest neighbors in the tree within the 4785 nucleotides, i.e. ST11 and ST30 for M6p and ST58 and ST59 for M11p, was 17 and 12 and eight and seven, respectively.

Based on phylogenetic analysis the third sample, M7p (indicated by $\bullet$, Fig. 1), did not fall into the clade of a given species but clustered between $B$. carolinensis and $B$. bissettii, two species widely distributed among the multiple hosts and vector ticks in the southeastern United States, although the node support was low (41 \%). The genetic distance analysis showed a value above the species threshold (0.017) compared with four B. carolinensis strains (0.021) and the majority of B. bissettii STs (0.022) (Table 1). However, for four B. bissettii STs (ST270 from California; ST271, ST275 and ST277 from Colorado) the genetic distance was just above the species threshold $(0.018$ and 0.019 ; species threshold 0.017) suggesting a close relationship of the M7p strain to $B$. bissettii. The species threshold of 0.017 for the MLSA System based on housekeeping genes was determined by comparison to data published earlier [18].

\section{Nucleotide sequence accession numbers}

Sequences determined in this study have been deposited into GenBank and given the indicated accession numbers (numbers for each isolate are given for sequenced genomic loci in the following order: $(\operatorname{lp} A, \operatorname{clp} X$, nifS, 


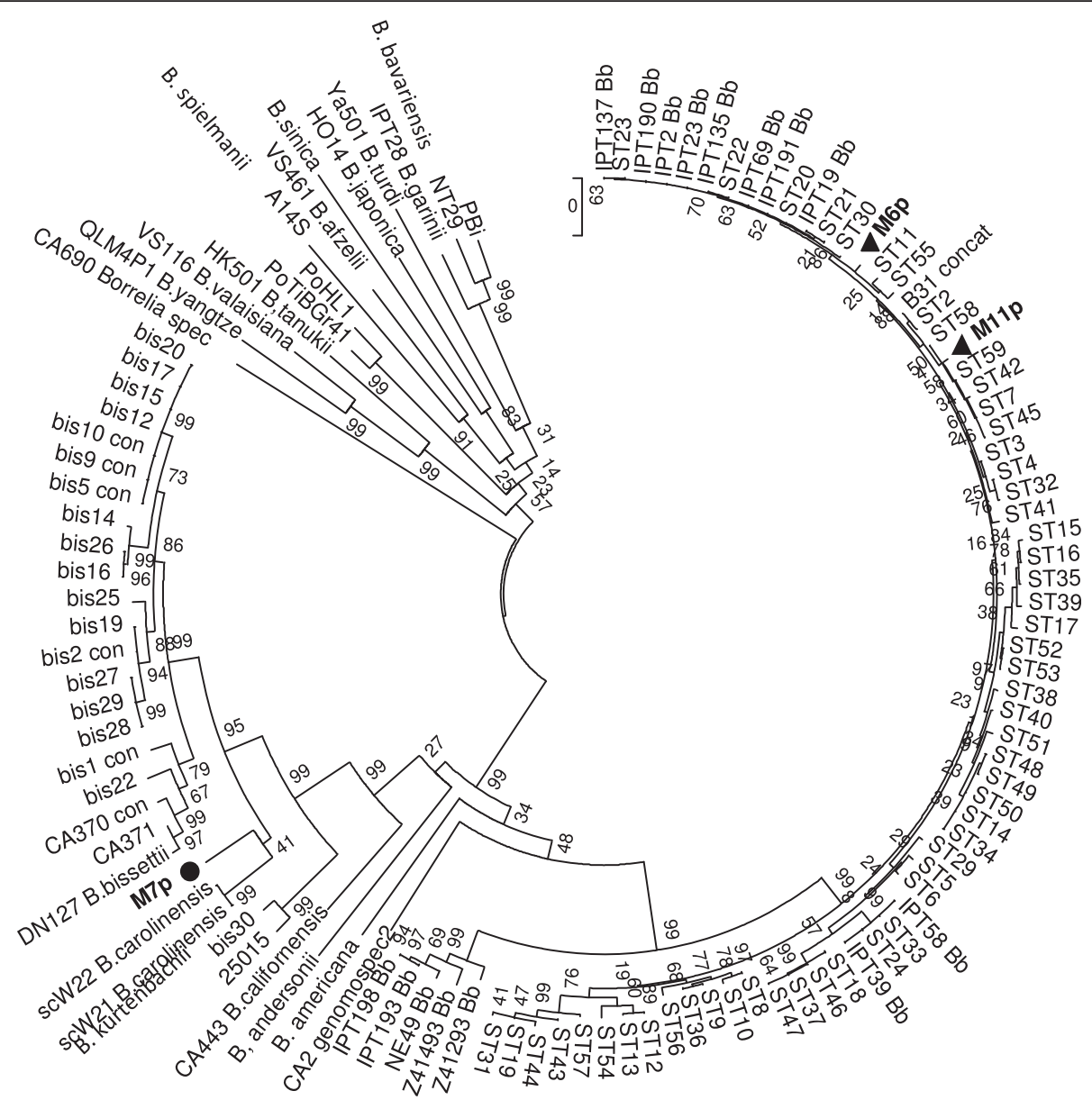

Fig. 1 A maximum likelihood tree was generated in MEGA5 [15] using concatenated sequences of eight housekeeping loci (clpA, clpX, nifS, pepX, pyrG, recG, rp/B and uvrA). The general time reversible model was employed and 500 bootstrap repeats used for estimation of node support. The large clade on the right hand site represents B. burgdorferi s.s. and two of the samples analyzed in this study (M6p and M11p, marked with fall into this clade. The third strain analyzed (M7p, $\bullet$ ) clusters between B. carolinensis and B. bissettii

pepX, pyrG, recG, rplB and uvrA) KM269425, KM2 69422, KM269428, KM269434, KM269443, KM269440, KM269437, KM269431 for M6p; KM269426, KM269423, KM269429, KM269435, KM269444, KM269441, KM269 438, KM269432 for M11p; KM269427, KM269424, KM26 9430, KM269436, KM269445, KM269442, KM269439, KM 269433 for M7p.

\section{Discussion}

In this study we investigated Borrelia strains that were isolated from residents in Southeastern US including one that was closely related to Borrelia bissettii. This is the first report of a $B$. bissettii being isolated from a human individual in the US. Until this research only B. burgdorferi s.s. strains were identified in samples of human origin in North America [10]. Today the number of reports that associate $B$. bissettii with human LB can be counted on one hand [8-10]. The lack of clinical evidence does not allow association of this spirochete species with specific clinical manifestations of the disease. However, earlier findings suggest that arthralgia is one of the disease manifestations caused by infection with $B$. bissettii in a group of LB diagnosed patients [9]. A single case of human endocarditis confirmed a known fact that LB spirochetes in general and B. bissettii in particular have a predilection for cardiac infection [8]. Those data are well correlated with a study of pathogenic potential of $B$. bissettii on murine model. The ability of $B$. bissettii strains to induce lesions in vertebrate host heart, femorotibial joint and bladder was confirmed 8 weeks after laboratory infection of mice with spirochete isolates [19].

Strains of B. bissettii reported thus far in the United States occur in rather moderate climatic regions of the western and southeastern parts of the country, but rarely in the northern region. B. bissettii is maintained in several enzootic transmission cycles that involve both human-biting "bridge" vectors (I. pacificus) and ticks with a strong host preference that do not bite humans (I. spinipalpis) in the west [20] and in the east coast (I. 
Table 1 Estimates of evolutionary divergence between sequences

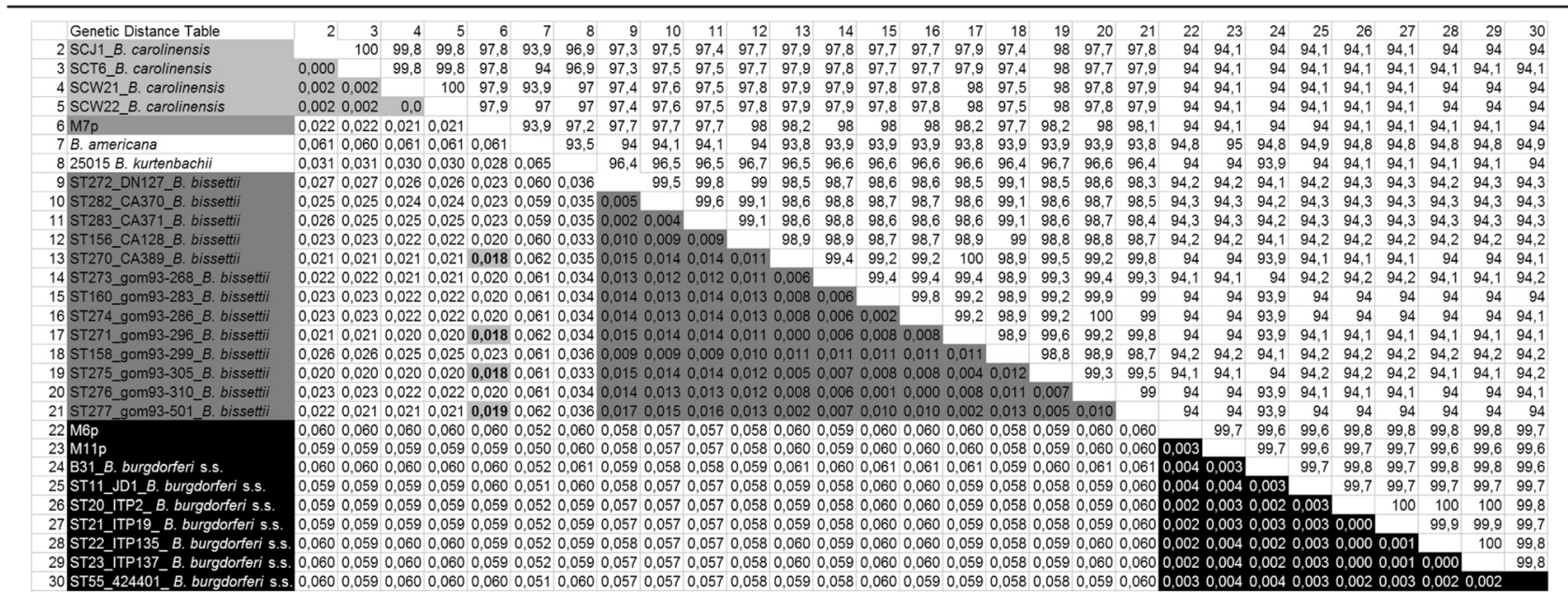

The numbers of base substitutions per site between sequences are shown in the lower left panel. In the upper right genetic similarities values are given. Strain M7p showed values that just exceeded the species threshold compared to some B. bissettii strains (bold numbers and shaded in light grey in column 6). The shaded regions in columns 2-4, 9-20 and 22-30 correspond to intraspecific divergence within genospecies (B. carolinensis, B. bissettii and B. burgdorferi sensu stricto, respectively). Analyses involved 29 nucleotide sequences and were conducted using the Kimura 2-parameter model [16]. Codon positions included were $1 s t+2 n d+3 r d+$ Noncoding. All positions containing gaps and missing data were eliminated. There were a total of 4785 positions in the final dataset. Evolutionary analyses were conducted in MEGA6 [17]

minor, I. affinis) [21]. B. bissettii strains were isolated from the dusky-footed wood rat (Neotoma fuscipes) and I. spinipalpis from California [22], and from the cotton mouse, the cotton rat, the eastern wood rat, I. scapularis, I. minor and I. affinis ticks from South Carolina and Georgia [23], [Oliver Jr., Rudenko, Golovchenko and Clark, unpublished observations]. B. bissettii was detected in co-infected isolates originated from skin biopsies of downy woodpecker (Dryobates pubescens), Carolina wren (Thryothorus ludovicianus) and northern water thrush (Parkesia noveboracensis), trapped in St. Catherines Island (GA, USA) in 1997 [Oliver Jr., Golovchenko and Rudenko, unpublished observations]. The records of naturally occurring infections in multiple species of rodents, birds, and Ixodes ticks indicate that $B$. bissettii is widely distributed in the United States and is not narrowly confined to particular vertebrate host or tick species. With such well-established population of $B$. bissettii in its area of distribution, with wide availability of multiple hosts and tick vectors, it is not surprising that residents of the west and east coast are exposed to this species.

The pathogenic potential of American strains of B. bissettii investigated on murine models was sufficient to induce disease, causing pathology within the heart, femorotibial joint and bladder in vertebrate host [19]. At the same time, European studies had shown that B. bissettii are able to infect humans and suggested they may be potentially human pathogenic $[8,9]$ although the published cases of human infections may be infrequent due to the rarity of $B$. bissettii-infections in human-biting host seeking Ixodes vectors [23-27].
Here, we present analysis of the first recovered live $B$. bissettii-related spirochete (strain M7p) from a resident of North America. The taxonomic status of this strain requires further investigation. Phylogenetic analysis resulted in clustering of M7p strain between two clades formed by $B$. carolinensis and B. bissettii with rather low node support (41\%). The value of genetic distance was above the species threshold compared with $B$. carolinensis and B. bissettii, which might mean that strain M7p represents a novel species from $B$. burgdorferi s. 1. complex. The species threshold of 0.017 for the MLSA based on housekeeping genes was determined by comparison to data published by Postic and colleagues earlier [18]. These authors compared DNA-DNA hybridization data with MLST data and used strains Z41293 and NE49 to set the threshold for species delineation in Borrelia. The same strains were used later [28] in the MLSA system based on housekeeping loci which allowed direct comparison of genetic distances between MLSA systems. It was found that strain NE49 that belongs to the same clade as Z41293 has a slightly different distance of 0.0183 . Thus, species thresholds are not determined by a strictly "fixed" genetic distance but maybe a little bit fuzzy. Another confirmation of this fact was present in a recent report about B.yangtzensis [29]. Two quite divergent populations of $B$. yangtzensis do exist with genetic distances above the species threshold [28]. These two divergent populations are "bridged" by a population that has genetic distances below the species threshold to both of these divergent populations, making this an example for a genetic continuum between divergent populations of a species. To analyze more strains similar to $\mathrm{M} 7 \mathrm{p}$ 
described in this study is very important to get a better picture how Borrelia species and populations are structured.

Isolation of live $B$. bissettii-like spirochetes from a human provides evidence that this species, in addition to $B$. burgdorferi sensu stricto, may cause human Lyme borreliosis in North America, possibly with clinical manifestations different from those related to B. burgdorferi s.s. infection [30].

\section{Competing interests}

The authors declare that they have no competing of interest

\section{Authors' contributions}

MG, MV and NR designed the experimental scheme, maintained strain cultivation and analysis, DNA purifications and amplifications, sequence analysis, results evaluation and drafted the manuscript. KC collected all human samples, evaluated results and drafted the manuscript. LG and JHO Jr secured financial support of the project, evaluated results and edited the manuscript. All authors read and approved the final version of the manuscript.

\section{Acknowledgments}

We are grateful to patients from Georgia and Florida and to their clinicians, for providing the samples. All credits for phylogenetic analysis and production of Fig. 1 and genetic distance analysis and Table 1 go to Dr. Gabriela Margos. This research was supported by European FP7 project 278976 ANTIGONE, GSU Foundation (USA) and institutional support RVO: 60077344 from Biology Centre, Institute of Parasitology (Czech Republic).

\section{Author details}

'Biology Centre Czech Academy of Sciences, Institute of Parasitology, Ceske Budejovice 37005, Czech Republic. ${ }^{2}$ The James H. Oliver Jr. Institute for Coastal Plain Sciences, Statesboro, Georgia Southern University, Georgia 30460-8056, USA. ${ }^{3}$ University of North Florida, Jacksonville, FL 32224, USA

${ }^{4}$ University of South Bohemia, Ceske Budejovice 37005, Czech Republic.

Received: 4 August 2015 Accepted: 1 February 2016

\section{Published online: 04 February 2016}

\section{References}

1. Wang G, van Dam AP, Schwartz I, Dankert J. Molecular typing of Borrelia burgdorferi sensu lato: taxonomic, epidemiological, and clinical implications. Clin Microbiol Rev. 1999;12:633-53.

2. Wang G, van Dam AP, Dankert J. Phenotypic and genetic characterization of a novel Borrelia burgdorferi sensu lato isolate from a patient with lymeborreliosis. J Clin Microbiol. 1999:37:3025-8.

3. Rijpkema SG, Tazelaar DJ, Molkenboer MJ, Noordhoek GT, Plantinga G, Schouls LM, et al. Detection of Borrelia afzelii, Borrelia burgdorferi sensu stricto, Borrelia garinii and group VS116 by PCR in skin biopsies of patients with erythema migrans and acrodermatitis chronica atrophicans. Clin Microbiol Infect. 1997;3: $109-16$.

4. Ryffel K, Péter O, Rutti B, Suard A, Dayer E. Scored antibody reactivity determined by immunoblotting shows an association between clinical manifestations and presence of Borrelia burgdorferi sensu stricto, B. garinii, B. afzelii, and B. Valaisiana in humans. J Clin Microbiol. 1999;37:4086-92.

5. Collares-Pereira M, Couceiro S, Franca I, Kurtenbach K, Schäfer SM, Vitorino $L$, et al. First isolation of Borrelia lusitaniae from a human patient. J Clin Microbiol. 2004;42:1316-8.

6. Picken RN, Cheng Y, Strle F, Picken MM. Patient isolates of Borrelia burgdorferi sensu lato with genomic and phenotypic similarities of strain 25015. J Infect Dis. 1996;174:1112-5.

7. Maraspin V, Ruzic-Sabljic E, Strle F. Lyme borreliosis and Borrelia spielmanii. Emerg Infect Dis. 2006;12:1177.

8. Rudenko N, Golovchenko M, Mokracek A, Piskunova N, Ruzek D, Mallatova $\mathrm{N}$, et al. Detection of Borrelia bissettii in cardiac valve tissue of a patient with endocarditis and aortic valve stenosis (Czech Republic). J Clin Microbiol. 2008;46:3540-3.

9. Rudenko N, Golovchenko M, Ruzek D, Piskunova N, Mallatova N, Grubhoffer L. Molecular detection of Borrelia bissettii DNA in serum samples from patients in the Czech Republic with suspected borreliosis. FEMS Microbiol Lett. 2009;292: 274-81.

10. Girard YA, Fedorova N, Lane RS. Genetic diversity of Borrelia burgdorferi and detection of B. bissettii-like DNA in serum of north-coastal California residents. J Clin Microbiol. 2011;49:945-54.

11. Rudenko N, Golovchenko M, Vancova M, Clark K, Grubhoffer L, Oliver Jr JH. Isolation of live Borrelia burgdorferi sensu lato spirochetes from patients with undefined disorders and symptoms not typical for Lyme diseases. Clin Microbiol Infect. 2015. doi:10.1016/j.cmi.2015.11.009.

12. Preac-Mursic V, Wilske B, Schierz G. European Borrelia burgdorferi isolated from humans and ticks culture conditions and antibiotic susceptibility. Zb Bakteriol Hyg A. 1986;263:112-8.

13. Rudenko N, Golovchenko M, Grubhoffer L, Oliver Jr JH. Borrelia carolinensis sp. nov., a new (14th) member of the Borrelia burgdorferi sensu lato complex from the southeastern region of the United States. J Clin Microbiol. 2009;47:134-41.

14. Margos G, Gatewood AG, Aanensen DM, Hanincová K, Terekhova D, Vollmer SA, et al. MLST of housekeeping genes captures geographic population structure and suggests a European origin of Borrelia burgdorferi. Proc Natl Acad Sci USA. 2008;105:8730-5.

15. Tamura K, Peterson D, Peterson N, Stecher G, Nei M, Kumar S. MEGA5 Molecular evolutionary genetics analysis using maximum likelihood evolutionary distance, and maximum parsimony methods. Mol Biol Evol. 2011:28:2731-9.

16. Kimura M. A simple method for estimating evolutionary rate of base substitutions through comparative studies of nucleotide sequences. J Mol Evol. 1980;16:111-20

17. Tamura K, Stecher G, Peterson D, Filipski A, Kumar S. MEGA6: Molecular Evolutionary Genetics Analysis version 6.0. Mol Biol Evol. 2013;30:2725-9.

18. Postic D, Garnier M, Baranton G. Multilocus sequence analysis of atypical Borrelia burgdorferi sensu lato isolates-description of Borrelia californiensis sp. nov., and genomospecies 1 and 2. Int J Med Microbiol. 2007;297:263-71.

19. Schneider BS, Schriefer ME, Ditrich G, Dolan MC, Morshed MG, Zeidner NS Borrelia bissettii isolates induce pathology in a murine model of disease. Vector Borne Zoonotic Dis. 2008;8:623-33.

20. Eisen L, Eisen RJ, Mun J, Salkeld DJ, Lane RS. Transmission cycles of Borrelia burgdorferi and B. bissettii in relation to habitat type in northwestern California. J Vector Ecol. 2009;34:81-91.

21. Oliver Jr JH. Lyme borreliosis in the southern United States: a review. J Parasitol. 1996:82:926-35.

22. Postic D, Ras NM, Lane RS, Hendson M, Baranton G. Expanded diversity among Californian Borrelia isolates and description of Borrelia bissettii sp. nov. (formerly Borrelia group DN127). J Clin Microbiol. 1998;36:3497-504.

23. Diuk-Wasser MA, Gatewood AG, Cortinas MR, Yaremych-Hamer S, Tsao J, Kitron $U$, et al. Spatiotemporal patterns of host-seeking Ixodes scapularis nymphs (Acari: Ixodidae) in the United States. J Med Entomol. 2006:43:166-76.

24. Margos G, Hojgaard A, Lane RS, Cornet M, Fingerle V, Rudenko N, et al. Multilocus sequence analysis of Borrelia bissettii strains from North America reveals a new Borrelia species, Borrelia kurtenbachii. Ticks Tick Borne Dis. 2010;1:151-8.

25. Margos G, Piesman J, Lane RS, Ogden NH, Sing A, Straubinger RK, et al. Borrelia kurtenbachii sp. nov., a widely distributed member of the Borrelia burgdorferi sensu lato species complex in North America. Int J Syst Evol Microbiol. 2014;64:128-30.

26. Rauter $\mathrm{C}$, Hartung T. Prevalence of Borrelia burgdorferi sensu lato species in Ixodes ricinus ticks in Europe: a metaanalysis. Appl Environ Microbiol. 2005 71:7203-16.

27. Lin T, Oliver Jr JH, Gao L, Kollars Jr TM, Clark KL. Genetic heterogeneity of Borrelia burgdorferi sensu lato in the southern United States based on restriction fragment length polymorphism and sequence analysis. J Clin Microbiol. 2001:39:2500-7.

28. Margos G, Vollmer SA, Kornet M, Garnier M, Fingerle V, Wilske B, et al. A new Borrelia species defined by multilocus sequence analysis of housekeeping genes. Appl Environ Microbiol. 2009;75:5410-6.

29. Margos G, Chu CY, Takano A, Jiang BG, Liu W, Kurtenbach K, et al. Borrelia yangtzensis sp. nov. a rodent associated species in Asia is related to $B$. valaisiana. Int J Syst Evol Microbiol. 2015. doi:10.1099/ijsem.0.000491.

30. Rudenko N, Golovchenko M, Grubhoffer L, Oliver Jr JH. Updates on Borrelia burgdorferi sensu lato complex with respect to public health. Ticks Tick Borne Dis. 2011:2:123-8. 